\title{
Phonological patterns (templates) in 5p deletion syndrome
}

\author{
Nina Gram Garmann, ${ }^{12}$ Kristian Emil Kristoffersen, ${ }^{3}$ Hanne Gram Simonsen ${ }^{2}$ \\ ${ }^{1}$ Oslo and Akershus University College of Applied Sciences, Norway; ${ }^{2}$ MultiLing, \\ University of Oslo, Norway; ${ }^{3}$ Frambu Resource Center for Rare Disorders, Norway
}

\section{Abstract}

Whole word phonological patterns (templates) in utterances produced by children with $5 \mathrm{p}$ deletion syndrome are analysed, addressing four questions: (1) Are children with $5 \mathrm{p}$ deletion syndrome able to generalise over words? (2) How does the template score of children with $5 p$ deletion syndrome relate to those of typically developing children and of the target language? (3) How do the template scores relate to other phonological measures, PCC and consonant variegation? (4) What can the relationship between template scores and phonological measures tell us about templates? Children with $5 \mathrm{p}$ deletion syndrome are able to generalise over words, some to a target like extent, others generalise more than expected for their age. The template scores relate to other phonological measures, with two exceptions. The exceptions indicate that the template score of a child with articulatory difficulties may reflect more detailed representations of the words in memory than she is able to express.

Keywords: Cri du chat syndrome, 5p deletion syndrome, templates, phonology, PCC-R, consonant variegation. 


\section{BACKGROUND}

\section{Phonological acquisition and templates}

There is a growing body of literature on whole-word phonology, claiming that children acquire the speech sounds of their ambient language(s) by learning words and generalise over them, eventually discovering the individual speech sounds (see Vihman and Croft, 2007 and Vihman \& Keren-Portnoy, 2013.) More recently, Vihman and colleagues (e.g. Vihman and Croft, 2007; Vihman and Velleman, 2000) have modelled this word-based process in terms of phonological templates, a theoretical construct akin to constructions as they are viewed within cognitive grammar.

Children's first words are influenced by the ambient language (the input), their babbling patterns and their phonetic repertoire (Vihman \& Velleman, 2000: 256). Children use their babbling patterns and phonetic skills to produce selected words that they already know (nearly) how to produce. Since their babbling patterns and phonetic skills are limited, the first words will conform to certain patterns, which are called templates.

In Vihman \& Croft (2007: 690-691), the use of templates is suggested to be a strategy to overcome limited memory capacities, production difficulties, as well as challenges in speech planning and processing. Vihman \& Vellemann (2000) argue that one way to deal with some of these problems in specific words is to overgeneralise the phonological patterns the child has already established when producing words that are too difficult for her. When a child extends her templates to convert unfamiliar words into phonetic patterns that the child already knows, the words are regarded as being adapted. According to Vihman and Velleman (2000: 256), then, templates may be understood as generalizations over known words, and adapted words represent extensions, or overgeneralizations, of the templates. 
As an illustration of the concept of templates, take the following example from a typically developing girl (Johanna) aged 1;4 from the corpus Garmann-Norwegian (Garmann, Hansen, Simonsen, \& Kristoffersen, in press) on CHILDES. The template in question is one of consonant harmony, with two syllables containing two identical consonants $\left(\mathrm{C}_{1} \mathrm{VC}_{1} \mathrm{~V}\right)$, a pattern that is common in the speech of typically developing children at this age (Vihman \& Croft, 2007: 691). Johanna uses the pattern both to produce selected words that fit her pattern, and to adapt words that do not fit her pattern. When she says ['pæpæ] for pappa 'daddy' and ['kakæ] for kake 'cake', she uses her consonant harmony template to select words that have consonant harmony in the target language. When she says ['pəpa] for lampa 'the lamp', however, she adapts the word into a consonant harmony production.

As already suggested, the use of templates is connected to the acquisition of speech sounds or segments. When the child acquires her first words, she will select words that contain segments that she already knows. When the child wants to express words containing segments she does not know how to articulate, she may overgeneralise templates, or phonological patterns, based on the sounds she has already mastered, to produce the new word. Later, when the child masters more consonants and vowels, the dependence on templates may fade. The use of templates, then, may be a powerful tool in word production in the period when a child wants to express more words than she is able to memorise, plan the execution of or actually produce (Vihman, Keren-Portnoy, Whitaker, Bidgood, \& McGillion, 2013).

As the use of templates is connected to the acquisition of speech sounds, one question is whether other phonological scores, like PCC-R (Per cent Consonants Correct - Revised, Shriberg, Austin, Lewis, McSweeny, and Wilson, 1997) and consonant variegation (Vihman et al., 2013), are sufficient when describing phonological knowledge at a specific time, or whether templates have additional explanatory value. In the literature, the use of templates is 
connected to the ability to generalise over actual phonetic patterns, that is, to make phonological abstractions (Vihman et al., 2013). The ability to generalise is normally seen as an advantage, and even a necessity, when acquiring language. Beckman, Munson, and Edwards (2007) argue that children with a phonological disorder lack the ability to generalise over individual tokens of words. Children with SLI, though, manage this level of abstraction, but have problems in generalising over phonotactic patterns in different words. This supports the view that children with language disorders have problems with generalizations and that generalizations are a good thing when acquiring language.

In contrast, Vihman et al. (2013) discovered that late talkers use more templates (in their analysis, they have a higher $\mathrm{P}($ attern)-score), than typically developing children at the 25-word point. ${ }^{1}$ Here, the children with language delay seem even better at generalising than the typically developing children. Dodd and McIntosh (2010) show that children with SLI are as good as typically developing children in rule abstraction skills, which may be compared to the abilities to generalise, but that the SLI children made 'wrong' or deviant generalizations. In total, it seems like the ability to generalise is not positive per se, it is also necessary to look at in which way and for how long you generalise over words.

Vihman et al. (2013) also found that a high template score was associated with slower advances in morphology. This adds to the proof that template use under certain conditions may be seen as a disadvantage when acquiring language. Vihman et al. (2013) admit that they do not quite understand how the ability to generalise over known words can be a disadvantage to language acquisition, and suggest that calculating the template score over only one recording may not be capturing the degree of template use in an optimal way. Moreover, they characterise a high template score at a high age as 'an extended period of holistic phonology',

\footnotetext{
1 The children were compared at the 25 -word point, i.e. when the children first produced 25 words in a 30 minutes' session.
} 
indicating that age in itself may be an explanatory factor. We interpret this as if holding on to a holistic strategy of language learning, and not being able to abstract segments and morphemes from the acquired lexicon at a relatively high age may be a sign of language delay.

Vihman et al. (2013) conclude that late talkers showed language delay, but not language disorder. The study of template use in children with varying degrees of language delay or disorder may reveal more about the notion of templates, and its usefulness in accounting for early generalizations. Provided that template use reflects more than phonological accuracy, e.g. the ability to make phonological generalizations, studies of templates in children with language disorders may also contribute to our knowledge of the nature of the acquisition of language in these populations. The present paper is a contribution to these areas, with an examination of utterances by a group of children with a rare genetic disorder associated with language problems, $5 \mathrm{p}$ deletion syndrome.

\section{$5 p$ deletion syndrome}

$5 \mathrm{p}$ deletion syndrome is a disorder caused by a partial deletion of genetic material on the short arm of chromosome 5. Even though the syndrome is rare, it is one of the most common disorders associated with a chromosomal deletion, with an incidence of 1 in 50,000 live births (Niebuhr, 1978). Some frequent clinical features are: a high-pitched cry in infancy and childhood (Sohner \& Mitchell, 1991; Sparks \& Hutchinson, 1980), malocclusion, hyper- and hypotonia and delayed motor development (Carlin, 1990), microcephaly (Niebuhr, 1978), mild-to-profound intellectual disability (Cornish, Bramble, Munir, \& Pigram, 1999), short attention span, hyperactivity, and a stereotypical, aggressive, and self-injurious behaviour pattern (Collins \& Cornish, 2002). 
According to the previous literature, between 23 and $50 \%$ of individuals with $5 \mathrm{p}$ deletion syndrome do not develop spoken language at all (Baird, Campbell, Ingram, \& Gomez, 2001; Carlin, 1990; Cornish \& Pigram, 1996; Wilkins, Brown, \& Wolf, 1980), and for those who acquire spoken language, receptive language skills appear to be better than expressive language skills (Cornish, Bramble, et al., 1999; Cornish \& Munir, 1998; Marignier et al., 2012). In the domain of phonetics and phonology, misarticulations and omissions are common (Cornish, Bramble, et al., 1999; Schlegel et al., 1967; Sparks \& Hutchinson, 1980). In two studies of consonant articulation in a smaller group of Norwegian children with $5 p$ deletion syndrome (Kristoffersen, 2004, 2008), various substitutions, omissions and cluster reductions were identified and described. The children participating in these two studies had small consonant inventories compared to those of far younger typically developing children acquiring Norwegian. The inventories also exhibited inter-subject variation both in number and type of consonants.

Kristoffersen (2008) examined error rates and error patterns in the consonant productions longitudinally in one Norwegian girl with 5p deletion syndrome. Error rates in single consonants were measured at four observation points $(4 ; 6,5 ; 9,7 ; 0$ and $9 ; 4)$ in terms of PCC-R (Shriberg et al., 1997). The PCC-R varied from $22.8 \%$ at 4;6 to $69 \%$ at 9;4, which is low as compared with both TD children the same age and children with SLI acquiring Swedish, a language closely related to Norwegian (Hansson \& Nettelbladt, 2002). These authors reported a PCC of $80.10 \%$ (SD 9.49) for the children with SLI in their group (age range 4;3 - 5;7; mean 4;11), and 98.69\% (SD 2.25) for a group of age-matched controls.

More recently, Kristoffersen, Garmann, and Simonsen (2014) studied a group of children diagnosed with $5 \mathrm{p}$ deletion syndrome, and found that these children to varying degrees had problems with producing the consonants of the target language. More specifically, the subjects had low PCC-R values, small consonant inventories with several 
recurrent types of deviant consonants (relative to the target language), inaccuracy in the realization of target phonemes and variable similarity to target words. This study clearly showed that children with this syndrome have deviant lexical representations mostly due to missing or substituted consonants. Thus, a further exploration of how children with this syndrome acquire words, would be to find out whether systematic phonological generalizations over words form part of the deviances that we find when we look at each consonant in isolation. An important aim of the present paper is to examine these phonological patterns in terms of the notion of templates.

In sum, individuals with $5 \mathrm{p}$ deletion syndrome have motoric problems, mild to profound mental disability, short attention span and low accuracy in consonant production, which all are problems that may influence language production as well as the representations of words and linguistic structures in the mind. We therefore wonder whether children with this syndrome use templates in a similar degree to typically developing children. Are children with mild to profound mental disability able to generalise over words at all? If so, what about the children with prevalent motoric problems: Is it possible that children with $5 p$ deletion syndrome have richer phonological representations in memory than their word productions would imply?

Against this background we address the following four questions:

(1) Are children with $5 p$ deletion syndrome able to generalise over words?

(2) How do the template scores of children with $5 p$ deletion syndrome relate to template scores of typically developing children as well as the template score of the target language? 
(3) How do the template scores of children with $5 p$ deletion syndrome relate to other phonological measures?

(4) What can the relationship between template scores and phonological measures tell us about templates?

Based on previous studies of templates in typical and atypical populations, and of language skills in individuals with $5 \mathrm{p}$ deletion syndrome, we expect that the subjects in our study use templates, and that they use it more extensively than expected for children aged four to twelve years. We also expect that the children with low PCC-R scores and low consonant variegation scores have higher template scores than children with high PCC-R scores and high consonant variegation scores. The argument is that children with few consonants and inaccurate pronunciations will use more templates, whereas children with accurate pronunciations and many consonants to a lesser degree need to rely on templates.

\section{METHOD}

\section{Subjects}

Eight subjects - two boys and six girls aged between four and 12 years - participated in the study. All were recruited through the Cri du chat family support group in Norway. One of the subjects $-\mathrm{A}-$ is different from the others, in the sense that she does not exhibit the motor problems commonly associated with the syndrome, and has only minor problems with language as compared to the other seven. A few descriptions of this atypical $5 p$ deletion syndrome exist in the literature (e.g. Cornish, Cross, Green, Willatt, \& Bradshaw, 1999), reporting language skills within the range of typical development. Subject A most probably belongs here. One of the subjects, C, speaks both Swedish and Norwegian, and some of her 
words are noticeably Swedish. She is included because Swedish and Norwegian are closely related languages, and the inclusion serves to expand the number of subjects.

Table 1 displays intelligibility ratings made by the second author based on the children's utterances during the experiment. The subjects are ranked from A to $\mathrm{H}$ based on intelligibility; ${ }^{2}$ within the same intelligibility rating the children are ranked from the oldest to the youngest.

Table 1.

TABLE 1. Participants' gender, age, number of unique utterances, utterance type, and intelligibility rated by the second author.

\begin{tabular}{|c|c|c|c|c|c|}
\hline Participant & Age & Gender & $\begin{array}{l}\text { Number of unique } \\
\text { utterances in task }\end{array}$ & $\begin{array}{l}\text { Utterance type } \\
\text { (in conversation) }\end{array}$ & Intelligibility \\
\hline A & $6 ; 4$ & $\mathrm{~F}$ & 88 & Multi-word & HIGH \\
\hline B & $6 ; 9$ & $\mathrm{~F}$ & 102 & Multi-word & MEDIUM \\
\hline $\mathrm{C}$ & $5 ; 4$ & $\mathrm{~F}$ & 136 & Multi-word & MEDIUM \\
\hline $\mathrm{D}$ & $12 ; 3$ & $\mathrm{~F}$ & 37 & One-word & LOW \\
\hline $\mathrm{E}$ & $10 ; 8$ & $\mathrm{~F}$ & 117 & Multi-word & LOW \\
\hline F & $10 ; 0$ & M & 86 & One-word & LOW \\
\hline G & $9 ; 2$ & M & 29 & One-word & LOW \\
\hline
\end{tabular}

\footnotetext{
${ }^{2}$ In Kristoffersen \& al. (2014) the recordings were also rated by five other persons, all with experience in rating and analysing disordered language production, to check the reliability of the (then) first author's ratings.
} 


$\begin{array}{llllll}\mathrm{H} & 4 ; 6 & \mathrm{~F} & 87 & \text { One-word } & \text { LOW }\end{array}$

The subjects were given a noun elicitation task, also used in two previous studies, Kristoffersen (2008) and Kristoffersen et al. (2014). As far as possible the items in the task were chosen so that the target words offered an opportunity to produce all consonant phonemes in initial, medial and final position in the target language. In the stimuli given to $G$ this was not possible. For details about the recording procedure, see Kristoffersen et al. (2014).

As a control group, we have four typically developing children, two girls and two boys, aged between 1;7 and 1;9. For each of them we picked a 30 minutes' video recording from the corpus Garmann-Norwegian on CHILDES. The recordings are the first recordings when the child says at least 50 words in a 30 minutes' session with his or her parents. The sessions consist of spontaneous speech and the recordings were done when the child was interacting with his or her parents.

\section{Transcription and data analysis}

The elicited utterances were narrowly transcribed in extIPA for disordered speech (Ball \& Müller, 2005) by a first transcriber, and then $20 \%$ of the utterances were independently transcribed by a second transcriber. ${ }^{3}$ Agreement between the two transcriptions was $71 \%{ }^{4}$ All measurements were based on the first transcriber's transcriptions.

\footnotetext{
${ }^{3}$ Their target dialects differed on a few points: the rhotic sounds were either apical or uvular. Furthermore, some of the target dialects have a series of retroflex consonants, others have not (see Kristoffersen (2007) for an outline of Norwegian). These differences have been taken into consideration in our analyses below.
} 
The consonant phones in these transcriptions were categorised with respect to manner and place of articulation, nasal vs. oral airstream mechanism, presence vs. absence of vocal cord vibration, and lateral vs. central oral airstream. Based on these transcriptions, template scores, PCC-R scores and consonant variegation scores were calculated.

Template scores in the data from the children with $5 \mathrm{p}$ deletion syndrome were calculated following the procedure in Vihman et al. (2013: 54-55) with some exceptions, noted below. The template scores build on template analyses of data from one test session for each child. First, all utterances are divided into one word items. If some words are repeated, one instance of each word type is picked for the analysis. The selection is based on either the more frequent articulation, or if none is more frequent than the other, the more target like pronunciation, or if none is neither more frequent nor more target like, any one instance is picked.

The words are then listed according to prosodic shape (i.e. CV or CVCV), and if some of the words within each prosodic shape have something in common, i.e. consonant harmony, starting with a labial, or ending in a fricative, these words are grouped together.

Next, the words are judged and marked as being either selected or adapted. Selected words are words that are articulated close to the target within the child's repertoire. That is, if the word has a medial [l], but the child does not produce any medial [l]s, a production without the medial [l] or with a substitution may still be judged target like.

Finally, the words are scored according to whether they are instances of a general or a specified, as well as stable, template, as well as being either selected or adapted. If a word is not part of a template, it is not given any points. Contrary to the analyses in Vihman et al.

\footnotetext{
${ }^{4}$ This figure may seem low in comparison with other studies. See Kristoffersen et al. (2014) for a discussion of possible explanations.
} 
(2013) all the words produced are included in the analyses, even if they receive only 0 points. If the word belongs to a template that is stable, i.e. if the template comprises $10 \%$ or more of the instances in the data, the word is given one point. If the template specifies more than the prosodic shape, e.g. consonant type, the word is given one additional point. Vowel length and stress placement are not considered as characteristics that specify a pattern beyond the prosodic shape. If the word is adapted to a template, it is given yet another additional point. This gives the following point system: Within a specified pattern which comprises at least 10 $\%$ of the words, selected words are given 2 points and adapted words 3 points. Within an unspecified pattern which comprises at least $10 \%$ of the words in the sample, selected words are given 1 point and adapted words 2 points. Within an unspecified pattern which comprises less than $10 \%$ of the words in the sample, all the words are given 0 points.

Following Vihman et al. (2013), we apply schema theory (Langacker, 1987: 68) to our analyses, and if a set of words may be specified instances of a more general schema which includes a smaller number of less specified words, the less specified words are considered to be instances of a more general schema. Hence, if a child produces 100 words, and 15 of them are $\mathrm{CVCV}$ words, of which 10 are consonant harmony words, those 10 consonant harmony words are considered to belong to a specified and stable pattern, whereas the other five are considered to be part of a stable (although unspecified) pattern because the ten consonant harmony words also can be regarded as part of the unspecified pattern.

Vihman et al. (2013: 55) includes only words that can be given at least one point in their $\mathrm{P}($ attern $)$ score analyses, and words that cannot be given a point are excluded. We have chosen to include all the words that the child produces, and give some words 0 points. When we include words that are given 0 points in the analyses, we reduce the template score, which again reflects a more target like production. Words are seldom given only 0 points, but one example would be if a child produces 100 words, and less than ten of them have three 
syllables or more. The words in this category are included in the analyses, but are not given any points, thereby decreasing the template score.

Following this procedure, the score is converted into percentages. On a scale from 0 $100 \%$, the value ' 0 ' indicates that all utterances are target-like, and no words follow a pattern that comprise at least $10 \%$ of the child's produced words, whereas the value ' 100 ' indicates that all utterances are adapted to specific and stable templates.

For the controls, the template score was calculated in the same way as for the children with $5 \mathrm{p}$ deletion syndrome. To make the measurements more comparable, we have transformed all the measurements into percentages.

Finally, as all languages are templatic to a certain degree (Vihman \& Croft, 2007), we have calculated the template score of target like pronunciation of the words in the data from participant E. We were first considering using the data from the participant with the highest number of unique words, 136 words (participant C). This participant, however, speaks both Norwegian and Swedish and produces quite a few characteristically Swedish words. We therefore analysed what would be the target like pronunciation of the data from the participant who produced the second highest number of unique words, 117 words (participant E). In this analysis, we took vowel length in the stressed syllable as well as word tone into account because these two features are distinctive in Norwegian. If the word belongs to a template that is stable, i.e. if the template comprises $10 \%$ or more of the instances in the data, the word is given one point. If the template specifies more than the prosodic shape (i.e. order of consonant and vowels as well as the number of syllables (like CVCV and CVC)) including vowel length and word tone, e.g. that the word is ending in a schwa, the word is given one additional point. All of the words in the target word sample are considered to be selected, i.e. target-like, so a word can be given a maximum of two points. 
The individual consonants in the utterances of the subjects with 5p deletion syndrome were also scored according to the measure Percent Consonants Correct Revised (PCC-R; Shriberg, Austin, Lewis, McSweeney, \& Wilson, 1997). PCC-R is designed for assessment of children with disordered speech and measures the extent to which the consonants in children's word productions agree with target pronunciations. Within this analysis, deletions and substitutions are deemed incorrect whereas distorted consonants are considered as correct. PCC-R was calculated independently by the first and second authors for two of the children, F and G. Interrater agreement was $95 \%$.

The consonant variegation score was calculated as follows (Vihman et al., 2013: 54): Each produced word was given points on a scale from one to three points. If the word contained only vowels, glides, or /h/, e.g. [a], [ja] or [ha], it was given one point. If it also contained one and only one consonant (e.g. [ma] or [mama]), it was given two points. If the word contained two or more different consonants, e.g. [kat], [site], it was given three points. The total scores were then converted into percentages.

\section{RESULTS}


In table 2 we present the template score for our eight subjects, along with their number of unique words:

TABLE 2. Participants' number of unique words

produced in task and template score

\begin{tabular}{cccc}
\hline ID & Number of & Template & \\
& unique words & score $(\%)$ & Template score category \\
\hline G & 29 & 67 & HIGH \\
D & 37 & 55 & MEDIUM \\
F & 86 & 51 & MEDIUM \\
C & 136 & 50 & MEDIUM \\
H & 87 & 46 & MEDIUM \\
A & 88 & 32 & LOW \\
B & 102 & 29 & LOW \\
E & 117 & & \\
\hline
\end{tabular}


We have divided the participants into three groups, based on their template scores. One participant, G, has a template score of 67 , which we rate as high. Next, we have four participants, D, F, C, H, who have template scores between 46 and 55 . We rate these as medium template scores. Finally, we have two participants, B and E, with a template score at 29 and 28 respectively, which we rate as low.

In table 3 we present the template scores for four typically developing Norwegian speaking children at their 50-word point, along with the numbers of unique words in the samples:

TABLE 3. Typically developing children's age, number of unique words produced in a recording session, and template score

\begin{tabular}{cccc}
\hline ID & Age & $\begin{array}{c}\text { Number of unique } \\
\text { words }\end{array}$ & Template \\
& $1 ; 7$ & 62 & 54 \\
sohanna & $1 ; 9$ & 72 & 53 \\
Olav & $1 ; 9$ & 63 & 52 \\
Mattis & $1 ; 8$ & 57 & 51 \\
Iben & & & \\
\hline
\end{tabular}

The typically developing children were aged between 1;7 and 1;9 when they first produced more than 50 words in a 30 minutes' session. At this point, all the children had template scores between 51 and 54, which can be compared to the template scores of the children with $5 \mathrm{p}$ deletion syndrome who had a medium template score.

In table 4 we present the template score for Norwegian as a target language: 
TABLE 4. Template score of Norwegian as a target language as measured in a sample with 117 words. 
The template score for the target language is $27 \%$, and can be compared to the three children with $5 p$ deletion syndrome who had low template scores. Only four phonological patterns came up as templates comprising more than $10 \%$ of the words in the sample. Two of the templates were generic: Monosyllabic words with a short vowel ([ $\left.{ }^{1} \mathrm{man}\right]$ 'man'), monosyllabic words with a long vowel ([ ${ }^{1}$ bi:l] 'car'). Two of the templates were specified: Monosyllabic words with a short vowel starting with a coronal ([ $\left.{ }^{1} \tan \right]$ 'tooth'), and bisyllabic words with word tone 2 and a long stressed vowel ending in -/ə/ ([ [:৩ə] 'lion').

Having compared the template scores of children with $5 p$ deletion syndrome with the template scores of typically developing children 1;7-1;9 years and a target like pronunciation of some of the data in the sample, we will now turn to compare the template scores of the children with $5 \mathrm{p}$ deletion syndrome and two other phonological measures: PCC-R and consonant variegation. The relationship between PCC-R and template scores is shown in table 5:

TABLE 5. Participants' template score,

PCC-R score and PCC-R category

\begin{tabular}{cccc}
\hline ID & Template & PCC-R & \\
& score (\%) & score & PCC-R category \\
\hline A & 32 & 93 & HIGH
\end{tabular}




\begin{tabular}{cccc} 
B & 29 & 83 & HIGH \\
E & 28 & 72 & HIGH \\
C & 50 & 52 & MEDIUM \\
D & 55 & 49 & MEDIUM \\
G & 67 & 35 & MEDIUM \\
H & 46 & 23 & LOW \\
F & 51 & 2 & LOW \\
\hline
\end{tabular}


As we can see, the three children with the lowest template scores, A, B and E, also have the highest PCC-R scores, as expected. Also as expected, two of the children with medium template scores, C, and D, have medium PCC-R scores. Participant G, with the highest template score, however, has a higher PCC-R score than participants $\mathrm{H}$ and $\mathrm{F}$, who have medium template scores but very low PCC-R scores.

Table 6 shows the relationship between template score and consonant variegation for each participant.

TABLE 6. Participants' template score,

consonant variegation score, and consonant variegation score category

\begin{tabular}{cccc}
\hline ID & Template & Consonant variegation & Consonant variegation \\
& score $(\%)$ & score $(\%)$ & score category \\
\hline E & 28 & 100 & HIGH \\
A & 32 & 88 & HIGH \\
B & 29 & 81 & HIGH \\
D & 55 & 67 & MEDIUM \\
C & 50 & 58 & MEDIUM \\
G & 67 & 42 & MEDIUM \\
H & 46 & 32 & LOW \\
F & 51 & 7 & \\
\hline & & & \\
\hline
\end{tabular}


As expected, the three children with the lowest template scores, A, B and E, have a higher consonant variegation score. The child with the highest template score, G, has a relatively low consonant variegation score, also as expected. Additionally, two of the participants with a medium template score, $\mathrm{D}$ and $\mathrm{C}$, also have medium consonant variegation scores. Again, participants $\mathrm{F}$ and $\mathrm{H}$ behave unexpectedly, with medium template scores but low consonant variegation scores.

\section{DISCUSSION AND CONCLUSION}

In this study, we investigated the use of whole word patterns - templates - in eight children with $5 \mathrm{p}$ deletion syndrome, based on a noun elicitation task. We compared their use of templates to that of younger, typically developing children and to the template score of the target language. Furthermore, we compared their template score with scores on two other phonological measures: PCC-R and consonant variegation.

Our first question was: Are children with $5 \mathrm{p}$ deletion syndrome able to generalise over words? Our answer to this question is yes, the children are able to generalise over words since they all have templates.

Our second question was: How does the template score of children with $5 p$ deletion syndrome relate to template scores of typically developing children as well as the template score of the target language? The children have template scores between 28 and 67, where the three lowest scores can be compared to the template score of a target like pronunciation of the words, and the four medium scores can be compared to the template scores of typically developing children aged between 1;7 and 1;9. Five of the eight subjects have higher scores 
than expected for their age, which indicates that these children have a language delay, and that they hold on to their holistic approach to lexical acquisition for a longer time than expected for typically developing children. These children, then, can make whole word generalizations, but have problems with analysing the recurring minor parts of the words, e.g. segments.

Our third question was: How do the template scores relate to other phonological measures? In most cases we have seen a clear relation between templates scores and the two other phonological measures, but in two of the cases, participants $\mathrm{F}$ and $\mathrm{H}$, there are less obvious direct relations.

One of the children with a low template score, participant A, has a target like pronunciation, which is expressed here by a low template score, a high PCC-R score and a high consonant variegation score. Participant B, also with a low template score, high PCC-R score and high consonant variegation score, has relatively target like pronunciation, only with some problems with /r/, occasionally also with /s/, and with some consonant clusters. Likewise, participant $\mathrm{E}$ has a low template score, a high PCC-R and a high consonant variegation score, but as she has larger problems with /r/ and consonant clusters and tends to do some fronting, she is less target like than participants $\mathrm{A}$ and $\mathrm{B}$, and more difficult to understand. These three children probably have rather accurate phonetic representations of the words in their memories, but differing degrees of motoric problems seem to make them more or less easy to understand.

The children with medium template scores come in two groups. The first group, including participants $\mathrm{C}$ and $\mathrm{D}$, have medium PCC-R and consonant variegation scores as expected. Their use of templates to a medium degree reflect their phonetic abilities. The second group, including participants $\mathrm{F}$ and $\mathrm{H}$, have medium template scores, but low PCC-R 
and consonant variegation scores. Although these children have few consonants, they are able to generalise over words. Participant F only expresses the correct number of syllables as well as quite a few of the vowels. He is not able to render any of the consonants, though. Participant $\mathrm{H}$ also mostly expresses the correct number of syllables, quite a few of the vowels, but also a few more consonants than participant F. It seems like their lexical representations are more detailed than what they are able to express, and their generalizations might first be due to articulatory limitations and second to memory and planning capabilities.

The participant with the highest template score, participant $\mathrm{G}$, was the participant who could not produce the regular words in the elicitation task. He mostly produces names of family, friends and assistants and this factor together with his relatively high PCC-R and consonant variegation scores may suggest that he has only a few words represented in his lexical memory, and that he has relatively detailed representations of these words.

Our fourth question was: What can the relationship between template scores and phonological measures tell us about templates? As suggested in the discussion of the third question, our answer to this fourth question is that in a picture naming task where some of the children have poor articulation, the template score can still say something about the detail of the representations of words in memory even if the child is not able to produce the same details. The templates give us access to the representations in memory in children with poor articulation.

For children with better articulation, the articulatory abilities seem to go along with the template score, and we will not be able to say if their representations are more detailed than what is revealed by their articulation. This indicates that the template score reflects both articulatory abilities and representations of words in memory, which becomes particularly clear to us when we look at the data from children with $5 p$ deletion syndrome. 
It was important that the data were collected by a naming task. If we had tried to analyse spontaneous speech data, it would be much harder to know which words the children with poor articulation were aiming at. We would therefore not have been able to conclude that the mental representations of words were richer than the children were able to show through their word productions.

The analyses have revealed, then, that children with $5 \mathrm{p}$ deletion syndrome are able to generalise over words, and some of them do it to a degree which characterises language delay. We have also seen that template scores reveal more than phonological measures do, and that templates reflect generalizations over words in memory as well as in production.

\section{ACKNOWLEDGEMENTS}

We thank the participants in the study, parents and children, for providing invaluable data for this piece of research, and Kristin Wium for transcription assistance. We wish to thank Marilyn Vihman for helpful and inspiring discussions about templates and how to calculate template scores, and the audiences at the Cognitive Summer Seminar in Troms $\varnothing$ 2012, ICPLA in Cork 2014 and Halifax 2016, as well as the reviewers of this paper for valuable comments and suggestions.

\section{STATEMENT OF INTEREST}

The authors report no declaration of interest. The work has been supported by The Research Council of Norway through its Centres of excellence scheme, project number 223265.

\section{REFERENCES}


Baird, S. M., Campbell, D., Ingram, R., \& Gomez, C. (2001). Young children with cri-duchat: Genetic, developmental, and behavioral profiles. Infant-Toddler intervention, 11(1), 1-14.

Ball, M. J., \& Müller, N. (2005). Phonetics for communication disorders. Mahwah, NJ: Lawrence Erlbaum Associates.

Beckman, M. E., Munson, B., \& Edwards, J. (2007). Vocabulary growth and the developmental expansion of types of phonological knowledge. In J. Cole \& J. I. Hualde (Eds.), Laboratory Phonology (Vol. 9). Berlin: Mouton de Gruyter.

Carlin, M. E. (1990). The improved prognosis in Cri-du-chat (5p-) syndrome. In W. I. Fraser (Ed.), Key issues in mental retardation research (pp. 64-73). London: Routledge.

Collins, M. S. R., \& Cornish, K. (2002). A survey of the prevalence of sterotypy, self-injury and aggression in children and young adults with Cri du Chat syndrome. Journal of Intellectual Disability Research, 46, 133-140.

Cornish, K., Bramble, D., Munir, F., \& Pigram, J. (1999). Cognitive functioning in children with typical cri du chat (5p-) syndrome. Developmental Medicine and Child Neurology, 41, 263-266.

Cornish, K., Cross, G., Green, A., Willatt, L., \& Bradshaw, J. (1999). A neuropsychologicalgenetic profile of atypical cri du chat syndrome: implications for prognosis. Journal of Medical Genetics, 36(7), 567.

Cornish, K., \& Munir, F. (1998). Receptive and expressive language skills in children with cri du chat syndrome. Journal of Communication Disorders, 31, 73-81.

Cornish, K., \& Pigram, J. (1996). Developmental and behavioural characteristics of cri du chat syndrome. Archives of disease in childhood, 75, 448-450.-448-450.

Dodd, B., \& McIntosh, B. (2010). Two-year-old phonology: Impact of input, motor and cognitive abilities on development. Journal of child language, 37, 1027-1046. 
Garmann, N. G., Hansen, P., Simonsen, H. G., \& Kristoffersen, K. E. (in press). Phonological characteristics of children's first words. In F. Chenu, S. Kern, \& F. Gayraud (Eds.), Proceedings from ELA 2012. Cambridge, UK: Cambridge University Press.

Hansson, K., \& Nettelbladt, U. (2002). Assessment of specific language impairment in Swedish. Logopedics Phoniatrics Vocology, 27(4), 146-154.

Kristoffersen, K. E. (2004). Consonant productions in three children with cri du chat syndrome. In B. E. Murdoch, J. Goozee, B.-M. Wehlan, \& K. Docking (Eds.), Proceedings of the 26th International Association of Logopaedics and Phoniatrics 2004. Brisbane: IALP.

Kristoffersen, K. E. (2007). Norwegian speech acquisition. In S. McLeod (Ed.), The International Guide to Speech Acquisition. Clifton Park: Thomson Delmar Learning. Kristoffersen, K. E. (2008). Consonants in Cri du chat syndrome: A case study. Journal of Communication Disorders, 41(3), 179-202.

Kristoffersen, K. E., Garmann, N. G., \& Simonsen, H. G. (2014). Consonant production and intelligibility in cri du chat syndrome. Clinical Linguistics \& Phonetics, 28(10), 769784. doi:10.3109/02699206.2014.904442

Langacker, R. W. (1987). Foundations of cognitive grammar, vol. 1, theoretical prerequisites. Stanford: Stanford University Press.

Marignier, S., Lesca, G., Marguin, J., Bussy, G., Sanlaville, D., \& Des Portes, V. (2012). Childhood apraxia of speech without intellectual deficit in a patient with cri du chat syndrome. European Journal of Medical Genetics, 55(6), 433-436.

Niebuhr, E. (1978). The cri du chat syndrome. Human genetics, 44(3), 227-275.

Schlegel, R., Neu, R., Carneiro, L., Reiss, J., Nolan, T., \& Gardner, L. (1967). Cri-du-chat syndrome in a 10 year old girl with deletion of the short arms of chromosome number 
5. Observations on dermatoglyphics, maxillo-mandibular measurements and sound spectrograms. Helvetica paediatrica acta, 22(1), 2.

Shriberg, L. D., Austin, D., Lewis, B. A., McSweeney, J. L., \& Wilson, D. L. (1997). The percentage of consonants correct (PCC) metric: Extensions and reliability data. Journal of Speech Language and Hearing Research, 40(4), 708-722.

Sohner, L., \& Mitchell, P. (1991). Phonatory and phonetic characteristics of prelinguistic vocal development in cri du chat syndrome. Journal of Communication Disorders, 24, 13-20.

Sparks, S., \& Hutchinson, B. (1980). Cri du chat: Report of a case. Journal of Communication Disorders, 13, 9-13.

Vihman, M. M., \& Croft, W. (2007). Phonological development: toward a 'radical' templatic phonology. Linguistics, 45, 628-725.

Vihman, M. M., Keren-Portnoy, T. (2013). The emergence of phonology. Whole-word approaches and cross-linguistic evidence. Cambridge, UK: Cambridge University Press.

Vihman, M. M., Keren-Portnoy, T., Whitaker, C., Bidgood, A., \& McGillion, M. (2013). Late talking toddlers: Relating early phonological development to later language advance. York Papers in Linguistics, 2(13), 47-68.

Vihman, M. M., \& Velleman, S. (2000). Phonetics and the origins of phonology. In N. Burton-Roberts, P. Carr, \& G. J. Docherty (Eds.), Phonological knowledge: Conceptual and empirical issues (pp. 305-339). Oxford: Oxford University Press.

Wilkins, L. E., Brown, J. A., \& Wolf, B. (1980). Psychomotor development in 65 homereared children with cri-du-chat syndrome. The Journal of Pediatrics, 97 (3), 401-405. 